\title{
Korean Dental Hygienists' Job Description Based on Systematic Review
}

\author{
Sun-Mi Kim', Eunsuk Ahn², Soo-Jeong Hwang ${ }^{3}$, Soon-Jeong Jeong ${ }^{4}$, Bo-Ra Kim5, \\ and Ji-Hyoung $\mathrm{Han}^{6, \dagger}$ \\ ${ }^{1}$ Department of Dental Hygiene, Wonkwang Health Science University, Iksan 54538, ${ }^{2}$ Department of Dental \\ Hygiene, Daejeon Institute of Science and Technology, Daejeon 35408, ${ }^{3}$ Department of Dental Hygiene, \\ College of Medical Science, Konyang University, Daejeon 35365, ${ }^{4}$ Department of Dental Hygiene \& Institute \\ of Basic Science for Well-Aging, Youngsan University, Yangsan 50510, ${ }^{5}$ Department of Dental Hygiene, College \\ of Medical and Health Sciences, Cheongju University, Cheongju 28503, ${ }^{6}$ Department of Dental Hygiene, Suwon \\ Science College, Hwaseong 18516, Korea
}

Background: Korean dental hygienists perform various tasks under the supervision of dentists in addition to the tasks listed in the law. Many meaningful studies have been conducted to determine the actual tasks of dental hygienists, but these studies did not show common results due to the differences in research methods or designs. Hence, this study aimed to review the reported data on the tasks of dental hygienists in Korea and to clarify them based on a systematic literature review.

Methods: For the literature search, the COre, Standard, and Ideal model presented by the National Library of Medicine was referenced. Seven databases were searched for literatures published in Korea, including PubMed, and Google Scholar. Of the 352 studies found using key words, titles, and abstracts, 46 were finally extracted based on the first and second exclusion criteria. After confirming the tasks of Korean dental hygienists in 46 literatures, 136 tasks were listed and calculated as appearance rate in the literature.

Results: The most common tasks in 46 studies were fluoride application (67.2\%), radiography (65.4\%), scaling (65.4\%), sealant $(60.7 \%)$, patient management and counseling (56.7\%), tooth-brushing education (52.2\%), impression taking with alginate (50.1\%), and making temporary crowns $(47.9 \%)$. The most mentioned tasks of dental hygienists in public health centers were fluoride application $(100 \%)$, sealant $(100 \%)$, oral health education $(71.4 \%)$, public oral health program evaluation $(71.4 \%)$, school fluoride mouth-rinsing program (71.4\%), water fluoridation (57.1\%), tooth-brushing education (57.1\%), school oral health programs (57.1\%), and public elderly oral health programs (57.1\%).

Conclusion: This study showed that Korean dental hygienists had 136 tasks by reviewing 46 related studies and that the main job of Korean dental hygienists was oral disease prevention including scaling, sealant, and fluoride application.

Key Words: Dental hygienists, Job description, Systematic review

\section{Introduction}

The oral health workforce in Korea is composed of dentists, dental hygienists, and dental technicians, and dental hygienists contribute to the improvement of the publics oral health by providing treatment that prevents oral diseases ${ }^{1)}$. Dental hygiene education in Korea began in 1965 and has made rapid progress since 2004, including the establishment of a 4-year curriculum ${ }^{2)}$ and the world's first doctoral course in dental hygiene ${ }^{3)}$. The tasks of dental hygienists were legally prescribed in the Medical Technicians Act of 1973 and include promotion of oral health and prevention of oral diseases; however, in reality, dental hygienists perform various tasks in dental clinics 
such as dental treatment assistance and business management support. In the task description reported in 2000, the tasks of dental hygienists are divided into six classifications: oral health education, preventive dental treatment, dental treatment cooperation, public health business, business management support, and professional improvement ${ }^{4)}$. As such, dental hygienists perform various tasks under the direction and supervision of dentists in addition to the tasks listed in the law. In the last decades, some dentists delegate their duties to dental hygienists to ensure the continuous performance of their tasks. However, it is not clear whether the task is legitimate or not $^{5)}$. In some cases, the tasks are not recognized as legitimate, and dental hygienists are oftentimes subject to administrative disposition. As a result, dental hygienists in Korea experience a feeling of deprivation along with a decrease in job satisfaction, and sometimes pursue a new career as they tend to have a negative perception about their job ${ }^{6-8)}$. Therefore, it is necessary to clearly confirm the tasks actually performed in the dental medical environment and conduct a job analysis accordingly. Based on this, the dental community should discuss the actual tasks performed by dental hygienists, and provision of institutional support is essential to ensure that the tasks performed are legitimate. In the meantime, many meaningful studies have been conducted to examine the actual tasks of dental hygienists in Korea, and many reference data have been obtained in order to establish an effective policy. These studies reflect the dental medical environment at the time of the investigation and propose the actual scope of responsibilities of dental hygienists. Nevertheless, it was not possible to reach a comprehensive conclusion as the finding of related research were consistent. Therefore, this study aimed to review the reported research data on the tasks, duties, and scope of tasks of dental hygienists in Korea and to clarify the tasks of dental hygienists based on a systematic literature review. Moreover, this study aimed to ensure that the tasks performed by dental hygienist are within their legal scope of practice and to our findings will serve as a basis for establishing policy agreements on the legal tasks of dental hygienists.

\section{Research Methods}

\section{Literature search}

For literature search, the COSI (COre, Standard, Ideal) model presented by the National Library of Medicine was referenced $^{9)}$. Based on the results of the discussions of researchers, PubMed (http://www.pubmed.gov), and

Table 1. Keywords Used in Literatures Search

\begin{tabular}{|c|c|c|}
\hline Priority & Primary keyword & Secondary keyword \\
\hline 1 & Dental hygienist task & Dental hygienist task \\
\hline 2 & Dental hygienist job & Dental hygienist job \\
\hline 3 & $\begin{array}{l}\text { Dental hygienist job } \\
\text { scope }\end{array}$ & $\begin{array}{l}\text { Dental hygienist task } \\
\text { scope }\end{array}$ \\
\hline 4 & $\begin{array}{l}\text { Dental hygienist task } \\
\text { scope }\end{array}$ & $\begin{array}{l}\text { Dental hygienist job } \\
\text { scope }\end{array}$ \\
\hline 5 & Role of dental hygienist & Role of dental hygienist \\
\hline 6 & Dental hygienist task area & $\begin{array}{l}\text { Dental hygienist medical } \\
\text { cooperation }\end{array}$ \\
\hline 7 & $\begin{array}{l}\text { Dental hygienist medical } \\
\text { cooperation }\end{array}$ & $\begin{array}{l}\text { Dental hygienist medical } \\
\text { assistance }\end{array}$ \\
\hline 8 & $\begin{array}{l}\text { Dental hygienist medical } \\
\text { assistance }\end{array}$ & $\begin{array}{l}\text { Dental hygienist core } \\
\text { competency }\end{array}$ \\
\hline 9 & $\begin{array}{l}\text { Dental hygienist core } \\
\text { competency }\end{array}$ & $\begin{array}{l}\text { Dental hygienist medical } \\
\text { personification }\end{array}$ \\
\hline 10 & $\begin{array}{l}\text { Dental hygienist medical } \\
\text { personification }\end{array}$ & $\begin{array}{l}\text { Dental hygienist medical } \\
\text { practice }\end{array}$ \\
\hline 11 & $\begin{array}{l}\text { Dental hygienist business } \\
\text { management support }\end{array}$ & $\begin{array}{l}\text { Dental } \\
\text { infection/Prevention of } \\
\text { infection }\end{array}$ \\
\hline 12 & $\begin{array}{l}\text { Dental hygienist medical } \\
\text { practice }\end{array}$ & $\begin{array}{l}\text { Dental hygienist } \\
\text { awareness }\end{array}$ \\
\hline 13 & $\begin{array}{l}\text { Dental } \\
\text { infection/prevention of } \\
\text { infection }\end{array}$ & Dental hygienist image \\
\hline 14 & Dental radiation safety & $\begin{array}{l}\text { Dental hygienist } \\
\text { recognition }\end{array}$ \\
\hline 15 & $\begin{array}{l}\text { Dental hygienist job } \\
\text { analysis }\end{array}$ & Dental medical dispute \\
\hline 16 & $\begin{array}{l}\text { Dental hygienist } \\
\text { awareness }\end{array}$ & $\begin{array}{l}\text { Dental hygienist } \\
\text { performance }\end{array}$ \\
\hline 17 & Dental hygienist image & \\
\hline 18 & $\begin{array}{l}\text { Dental hygienist } \\
\text { recognition }\end{array}$ & \\
\hline 19 & Medical law revision & \\
\hline 20 & Dental medical dispute & \\
\hline 21 & Oral health education & \\
\hline 22 & Health promotion & \\
\hline 23 & $\begin{array}{l}\text { Preventive dental } \\
\text { treatment }\end{array}$ & \\
\hline 24 & $\begin{array}{l}\text { Dental hygienist } \\
\text { performance }\end{array}$ & \\
\hline
\end{tabular}


Google Scholar (https://scholar.google.co.kr), which is a foreign database that can be used for general literature search; another five databases, where literature published in Korea can be searched; and their own journal sites related to dental hygiene that are recognized in Korea, were used for the study. Selected domestic databases include Korean academic information (http://kiss.kstudy. com), academic research information service (http://www. riss.kr), paper search (http://www.papersearch.net), Academic Education Center (https://www.earticle.net), DBpia (http:// www.dbpia.co.kr), Journal of Dental Hygiene Science (http://www.jkdhs.org), and Korean Dental Hygiene Society (http://www.jksdh.or.kr). In order to select the appropriate keywords to be used in the literature search, some previous studies were analyzed, and the first keyword was selected through a meeting with all of the researchers. After searching the literature using the primary keyword, the secondary keyword was extracted through deletion or correction of duplicates when searching the literature (Table 1).

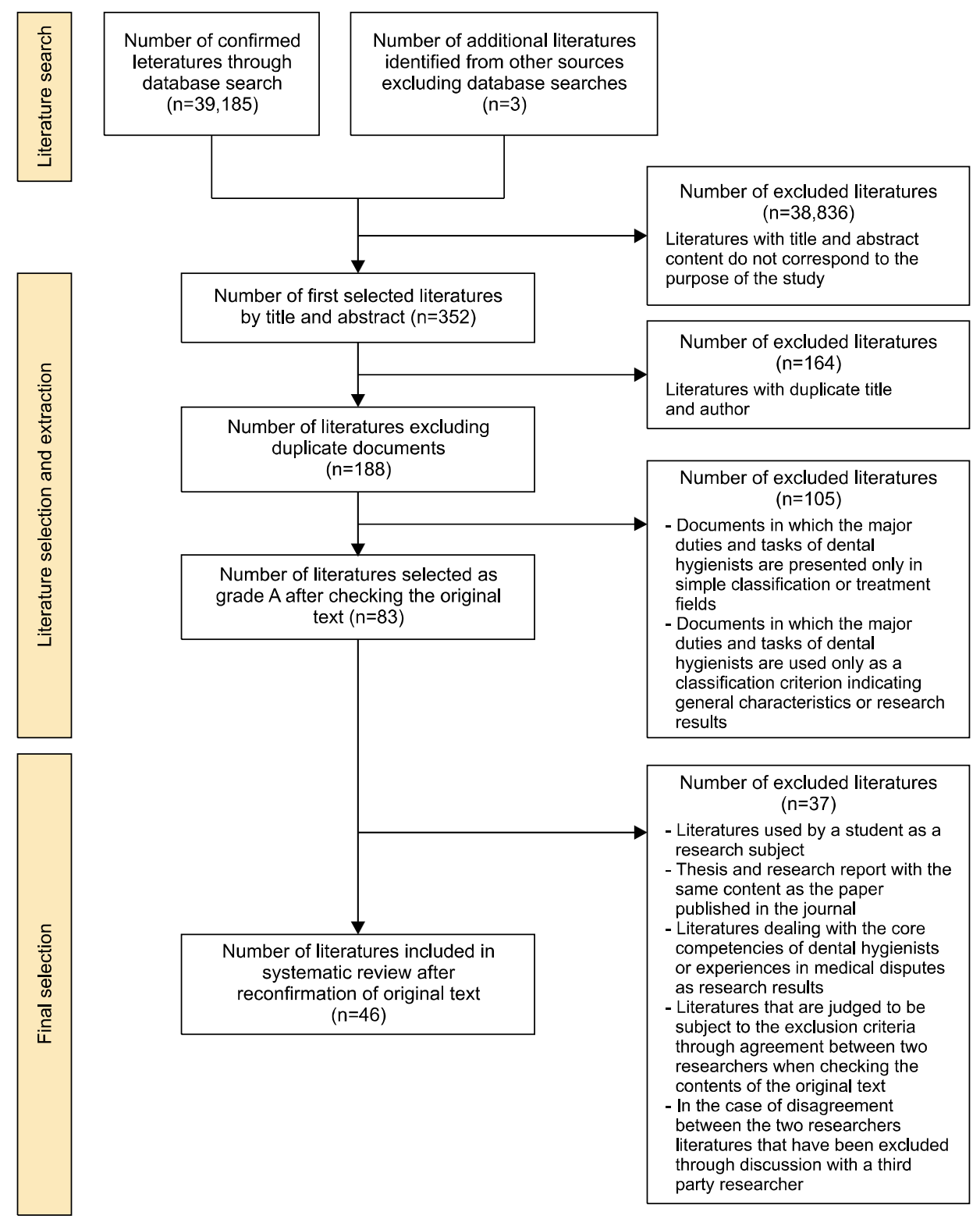

Fig. 1. Process of selection and exclusion of papers modified from PRISMA 2009 flow diagram ${ }^{10)}$. 


\section{Paper extraction}

The literature search was only conducted from 2000 to 2019 to reflect the recent trend, and a total of 39,188 articles were searched. The numbers of searches by keyword were as follows: 18,024 (46.0\%) for dental hygienist role, 4,635 (11.8\%) for dental hygienist tasks, 2,508 (6.4\%) for dental hygienist Jobs, and 2,368 (6.0\%) for dental hygienist image. After screening the titles and abstracts of each article, those that did not match the research purpose were initially excluded; as a result, only 352 articles were extracted. Afterward, duplicate literatures and literatures that did not provide sufficient data on the tasks of dental hygienists were excluded during the 2nd and 3rd exclusion processes. Over 83 studies were extracted during the third selection process, and 37 studies that were judged to be insufficiently related to this study after screening the original text and reviewing the consensus of the researchers were further excluded (Fig. $1)^{10)}$. A total of 46 literatures were finally selected (Table 2), of which 19 were conducted before 2010 and 27 after 2010. Of the total selected literature, 22 were journals (47.8\%), 19 were dissertations (41.3\%), and 5 were reports (10.9\%).

\section{Presentation of data}

After confirming the tasks of Korean dental hygienists in 46 literatures, each task was listed in specific terms. Then, a mark was applied when the dental hygienist's tasks appeared in each article, and the appearance rate in the entire literature was calculated. Most dental hygienists in Korea work in dental clinics, but more than 1,500 dental hygienists work in public health centers and implement public oral health programs. Therefore, it is necessary to classify the tasks of dental hygienists working in dental clinics and those working in public health centers. Of the 46 studies that were finally selected, 39 evaluated the tasks of clinicians, 6 evaluated the tasks of a public health center, and 1 evaluated the tasks of a clinical and dental hygienist at the same time. Therefore, based on 40 studies, including the tasks of a clinical dental hygienist and 7 literatures including the tasks of a health center dental hygienist, the rates of the appearance of the tasks of a clinical dental hygienist and a public health center dental hygienist were presented, respectively.

\section{Results}

A systematic literature review found that dental hygienists performed up to 136 tasks (Table 3). The most common tasks reported in 46 studies were fluoride application (67.2\%), radiography (65.4\%), scaling (65.4\%), sealant $(60.7 \%)$, patient management and counseling (56.7\%), tooth-brushing education (52.2\%), and impression taking (alginate) $(50.1 \%)$. Preventive treatment-related tasks such as fluoride application, scaling, and sealant appeared more frequently, and oral health education tasks such as patient management and counseling, and tooth-brushing education also frequently appeared. In addition, studies related to general dental treatments such as alginate impression taking, temporary crowns making, setting of temporary crowns, and application of temporary filling were investigated in the literature. More than half of the 40 studies studied on clinical dental hygienists included scaling, internal/external radiography, temporary crowns making, sealant, tooth-brushing education, and temporary filling as the tasks of dental hygienists. The task with the highest frequency was scaling $(74.4 \%)$, followed by Intraoral radiography and panorama taking (71.8\%), patient management and counseling $(64.1 \%)$, and fluoride application $(61.5 \%)$. In more than half of papers, impression taking (alginate, 56.4\%), temporary crowns making (53.8\%), setting of temporary crowns $(53.8 \%)$, sealant $(53.8 \%)$, tooth-brushing education $(51.3 \%)$, and temporary filling (51.3\%) were reported as the tasks of dental hygienists. In seven studies on dental hygienists working in public health centers, fluoride application and sealant accounted for $100 \%$ of the tasks performed by dental hygienists, oral health education, school fluoride mouth-rinsing program, public oral health program evaluation accounted for $71.4 \%$, and tooth-brushing education, education to use oral health products, oral examination, public elderly oral health program, water fluoridation, school water fluoridation, school oral health program accounted for $57.1 \%$. Dental hygienists working in public health centers perform 67 out of 136 tasks, and they are conducting oral disease prevention tasks as well 
Table 2. The 46 Literatures Selected Finally for Tasks Analysis of Korean Dental Hygienists

\begin{tabular}{|c|c|c|c|c|c|}
\hline Number & $\begin{array}{l}\text { Main } \\
\text { author }\end{array}$ & $\begin{array}{l}\text { Publication } \\
\text { year }\end{array}$ & Title & Publisher or journal & URL \\
\hline 1 & JH Sung & 2000 & $\begin{array}{l}\text { A study on the roles of dental } \\
\text { hygienists at the public health } \\
\text { centers in Korea }\end{array}$ & $\begin{array}{l}\text { Journal of Korean } \\
\text { Academy of Dental } \\
\text { Hygiene }\end{array}$ & $\begin{array}{l}\text { http://kadh.or.kr/index.php?mid=board_ } \\
\text { CnLa25\&category=2291\&document_sr } \\
\text { l=2330\&listStyle=list }\end{array}$ \\
\hline 2 & MY Hwang & 2002 & $\begin{array}{l}\text { A study on the Curriculums for } \\
\text { the Department of Dental } \\
\text { Hygiene - Based on the Tasks } \\
\text { of Hygienists and the Couses } \\
\text { For the National Qualification } \\
\text { Ecamination }\end{array}$ & $\begin{array}{l}\text { Bulletin of Dongnam } \\
\text { Health University }\end{array}$ & $\begin{array}{l}\text { https://kmbase.medric.or.kr/Main.aspx?d } \\
\text { =KMBASE\&i=0974620020200020123 } \\
\text { \&m=VIEW }\end{array}$ \\
\hline 3 & JH Yoo & 2003 & $\begin{array}{l}\text { Usefulness of dental hygiene } \\
\text { education curriculum in the } \\
\text { public oral health service } \\
\text { performance }\end{array}$ & Yonsei University & $\begin{array}{l}\text { https://ir.ymlib.yonsei.ac.kr/bitstream/22 } \\
\text { 282913/128826/1/T008015.pdf }\end{array}$ \\
\hline 4 & EH Kim & 2004 & $\begin{array}{l}\text { A study on the work of dental } \\
\text { hygienists and nurse aides }\end{array}$ & Inje University & $\begin{array}{l}\text { http://imgsvr.riss4u.net/contents3/td_con } \\
\text { tents8/09865/664/09865664.pdf }\end{array}$ \\
\hline 5 & JR Park & 2004 & $\begin{array}{l}\text { A research on the expansion of } \\
\text { dental hygienists' work }\end{array}$ & $\begin{array}{l}\text { Korean Dental } \\
\text { Hygienists Association }\end{array}$ & https://www.kdha.or.kr/news/report.aspx \\
\hline 6 & MH Lim & 2004 & $\begin{array}{l}\text { A Study on Dentists' } \\
\text { Awareness About Dental } \\
\text { Hygienists' Works and Duty } \\
\text { Division }\end{array}$ & $\begin{array}{l}\text { Journal of Korean } \\
\text { Academy of Dental } \\
\text { Hygiene }\end{array}$ & $\begin{array}{l}\text { http://kadh.or.kr/index.php?mid=board_ } \\
\text { CnLa25\&category=2293\&document_sr } \\
\text { l=2385\&listStyle=list }\end{array}$ \\
\hline 7 & PK Cho & 2004 & $\begin{array}{l}\text { An analsysis of the job } \\
\text { performance in operative } \\
\text { restoration by dental } \\
\text { hygienists }\end{array}$ & $\begin{array}{l}\text { Journal of Korean } \\
\text { Academy Dental } \\
\text { Hygiene Education }\end{array}$ & $\begin{array}{l}\text { http://www.jksdh.or.kr/view/CHOSAB-0 } \\
\text { 4-2-11.pdf }\end{array}$ \\
\hline 8 & KJ Won & 2005 & $\begin{array}{l}\text { A empirical study on job } \\
\text { education of dental health } \\
\text { administrative personnel }\end{array}$ & $\begin{array}{l}\text { Gangneung National } \\
\text { University }\end{array}$ & $\begin{array}{l}\text { http://www.riss.kr/search/detail/DetailVi } \\
\text { ew.do?p_mat_type=be54d9b8bc7cdb09 } \\
\text { \&control_no=9af4767c6610fe } 8 \mathrm{~d}\end{array}$ \\
\hline 9 & SY Kim & 2006 & $\begin{array}{l}\text { (A) Study on the curriculum for } \\
\text { dental hygienist education }\end{array}$ & Dankook University & $\begin{array}{l}\text { http://www.riss.kr/search/detail/DetailVi } \\
\text { ew.do?p_mat_type=be54d9b8bc7cdb09 } \\
\text { \&control_no=a1fd5bff7709a242ffe0bd } \\
\text { c3ef48d419 }\end{array}$ \\
\hline 10 & SS Bae & 2006 & $\begin{array}{l}\text { Development of role model for } \\
\text { dental hygienist in the changed } \\
\text { environment medical service }\end{array}$ & Yonsei University & $\begin{array}{l}\text { http://www.riss.kr/search/detail/DetailVi } \\
\text { ew.do?p_mat_type=be54d9b8bc7cdb09 } \\
\text { \&control_no=b90c1a558a6d1a6dffe0b } \\
\text { dc3ef48d419 }\end{array}$ \\
\hline 11 & YS Ahn & 2006 & $\begin{array}{l}\text { Comparison of job description } \\
\text { and turnover trend among } \\
\text { dental hygienists and assistant } \\
\text { nurses in dental clinics }\end{array}$ & $\begin{array}{l}\text { Journal of Korean } \\
\text { Academy of Oral } \\
\text { Health }\end{array}$ & $\begin{array}{l}\text { http://210.101.116.28/W_files/kiss61/21 } \\
\text { 101049_pv.pdf }\end{array}$ \\
\hline 12 & Shin & 2007 & $\begin{array}{l}\text { A study on the estimation of the } \\
\text { number of dental hygienist and } \\
\text { their practice }\end{array}$ & $\begin{array}{l}\text { Journal of Dental } \\
\text { Hygiene Science }\end{array}$ & $\begin{array}{l}\text { https://kmbase.medric.or.kr/KMID/1023 } \\
420070070010025\end{array}$ \\
\hline 13 & SH Shim & 2007 & $\begin{array}{l}\text { A study on the job awareness of } \\
\text { dental hygienists and their job } \\
\text { performance }\end{array}$ & $\begin{array}{l}\text { Journal of Korean } \\
\text { Society of Dental } \\
\text { Hygiene }\end{array}$ & $\begin{array}{l}\text { http://kiss.kstudy.com/thesis/thesis-view. } \\
\text { asp?key=2853967 }\end{array}$ \\
\hline 14 & YA Cheng & 2007 & $\begin{array}{l}\text { A predictive study on the role } \\
\text { and function of the dental } \\
\text { hygienist in Taiwan }\end{array}$ & $\begin{array}{l}\text { International Journal of } \\
\text { Dental Hygiene }\end{array}$ & $\begin{array}{l}\text { https://doi.org/10.1111/j.1601-5037.2007. } \\
\text { 00236.x }\end{array}$ \\
\hline 15 & YJ Kang & 2008 & $\begin{array}{l}\text { An analysis of the completion } \\
\text { and recognition of } \\
\text { preceptorship course for } \\
\text { fresh-dental hygienists }\end{array}$ & $\begin{array}{l}\text { Gyeongsang National } \\
\text { University }\end{array}$ & $\begin{array}{l}\text { https://dcollection.gnu.ac.kr/public_reso } \\
\text { urce/pdf/000000011540_20201116132 } \\
\text { 226.pdf }\end{array}$ \\
\hline
\end{tabular}


Table 2. Continued 1

\begin{tabular}{|c|c|c|c|c|c|}
\hline Number & $\begin{array}{l}\text { Main } \\
\text { author }\end{array}$ & $\begin{array}{c}\text { Publication } \\
\text { year }\end{array}$ & Title & Publisher or journal & URL \\
\hline 16 & YS Kim & 2008 & $\begin{array}{l}\text { A study on the current state and } \\
\text { weight of dental hygienists' } \\
\text { works }\end{array}$ & $\begin{array}{l}\text { Journal of Korean } \\
\text { Society of Dental } \\
\text { Hygiene }\end{array}$ & $\begin{array}{l}\text { http://www.jksdh.or.kr/view/CHOSAB-0 } \\
\text { 8-3-08.pdf }\end{array}$ \\
\hline 17 & SS Park & 2008 & $\begin{array}{l}\text { A Study on Elaboration of } \\
\text { Dental Hyenists' Participated } \\
\text { Items in Implant Treatment }\end{array}$ & $\begin{array}{l}\text { Gyeongsang National } \\
\text { University }\end{array}$ & $\begin{array}{l}\text { http://www.riss.kr/search/detail/DetailVi } \\
\text { ew.do?p_mat_type=be54d9b8bc7cdb09 } \\
\text { \&control_no=8121c9e1bb4df2d4ffe0bd } \\
\text { c3ef48d419 }\end{array}$ \\
\hline 18 & MS Wang & 2008 & $\begin{array}{l}\text { The roles of dental hygienist in } \\
\text { Korea }\end{array}$ & Dankook University & $\begin{array}{l}\text { http://lib.dankook.ac.kr/dcollection/publi } \\
\text { c_resource/pdf/000000032005_202011 } \\
\text { 16133237.pdf }\end{array}$ \\
\hline 19 & YH Cho & 2008 & $\begin{array}{l}\text { Recognition of the job } \\
\text { jurisdiction and job } \\
\text { satisfaction of the dental } \\
\text { hygienists and nursing aids }\end{array}$ & Kyungwon University & $\begin{array}{l}\text { http://gachon.dcollection.net/public_reso } \\
\text { urce/pdf/000000305245_20201116141 } \\
\text { 741.pdf }\end{array}$ \\
\hline 20 & YN Park & 2010 & $\begin{array}{l}\text { Dental office manager current } \\
\text { conditions by scale of Korean } \\
\text { dental clinic }\end{array}$ & $\begin{array}{l}\text { The Journal of the Korea } \\
\text { Contents Association }\end{array}$ & $\begin{array}{l}\text { https://www.dbpia.co.kr/journal/articleD } \\
\text { etail?nodeId=NODE01543074\&nodeId } \\
\text { =NODE01543074\&language=ko_KR }\end{array}$ \\
\hline 21 & JH Park & 2010 & $\begin{array}{l}\text { Dentists' opinions for dental } \\
\text { hygienists' roles in Korea }\end{array}$ & $\begin{array}{l}\text { Journal of Korean } \\
\text { Academy of Oral } \\
\text { Health }\end{array}$ & $\begin{array}{c}\text { http://journaleng.kstudy.com/ISS_Detail. } \\
\text { asp?key=2844604\&tname=kiss2002\&c } \\
\text { ode=YqldZWtoSqVtJTNENnAlOCUm } \\
\text { N/B\%20Z\%20xLJTNEVHJpZQ== }\end{array}$ \\
\hline 22 & YJ Song & 2010 & $\begin{array}{l}\text { The working range analysis for } \\
\text { the dental hygienists' } \\
\text { education program and legal } \\
\text { system improvement }\end{array}$ & Yonsei University & $\begin{array}{l}\text { https://ir.ymlib.yonsei.ac.kr/handle/2228 } \\
\text { 2913/137418 }\end{array}$ \\
\hline 23 & MO Ha & 2010 & $\begin{array}{l}\text { A study on the job analysis of } \\
\text { dental hygienist in Gwangju }\end{array}$ & $\begin{array}{l}\text { Journal of Korean } \\
\text { Society of Dental } \\
\text { Hygiene }\end{array}$ & $\begin{array}{l}\text { http://kiss.kstudy.com/thesis/thesis-view. } \\
\text { asp?key=2884088 }\end{array}$ \\
\hline 24 & GA Kang & 2011 & $\begin{array}{l}\text { A Study of Influential Factors } \\
\text { on Dental Hygienists' Duties } \\
\text { and their Job Satisfaction }\end{array}$ & $\begin{array}{l}\text { Journal of Korean } \\
\text { Academy of Dental } \\
\text { Hygiene }\end{array}$ & $\begin{array}{l}\text { http://kadh.or.kr/index.php?mid=board_ } \\
\text { CnLa25\&category=2274\&document_sr } \\
\text { l=2640\&listStyle=list }\end{array}$ \\
\hline 25 & HJ Noh & 2011 & $\begin{array}{l}\text { A Study on the Realization of } \\
\text { Work of Dental Hygienists in } \\
\text { Public Health Centers }\end{array}$ & $\begin{array}{l}\text { Korean Dental } \\
\text { Hygienists Association }\end{array}$ & https://www.kdha.or.kr/news/report.aspx \\
\hline 26 & JR Park & 2012 & $\begin{array}{l}\text { The 2nd Job Analysis Study of } \\
\text { Dental Hygienist }\end{array}$ & $\begin{array}{l}\text { Korea Health Personnel } \\
\text { Licensing Examination } \\
\text { Institute }\end{array}$ & $\begin{array}{l}\text { https://kiss.kstudy.com/public/public3-ar } \\
\text { ticle.asp?key=60013748 }\end{array}$ \\
\hline 27 & YS Cho & 2011 & $\begin{array}{l}\text { Review: Clinical Dental } \\
\text { Hygiene Education and } \\
\text { Practice based on Dental } \\
\text { Hygiene Process }\end{array}$ & $\begin{array}{l}\text { Journal of Dental } \\
\text { Hygiene Science }\end{array}$ & $\begin{array}{l}\text { http://kiss.kstudy.com/thesis/thesis-view. } \\
\text { asp?key=2935724 }\end{array}$ \\
\hline 28 & YL Kwak & 2013 & $\begin{array}{l}\text { Dentists' awareness of Dental } \\
\text { hygienists' work }\end{array}$ & KyungHee University & $\begin{array}{l}\text { http://khu.dcollection.net/public_resourc } \\
\text { e/pdf/200000063746_20201116154712. } \\
\text { pdf }\end{array}$ \\
\hline 29 & JH Kwon & 2013 & $\begin{array}{l}\text { The management efficiency } \\
\text { between the oral health clinic } \\
\text { and oral health center system }\end{array}$ & Dankook University & $\begin{array}{l}\text { http://lib.dankook.ac.kr/dcollection/publi } \\
\text { c_resource/pdf/000000176872_202011 } \\
\text { 16154906.pdf }\end{array}$ \\
\hline 30 & SY Ahn & 2013 & $\begin{array}{l}\text { Some dental hygienist by career } \\
\text { expectations regarding clinical } \\
\text { work done in the field of } \\
\text { business studies }\end{array}$ & $\begin{array}{l}\text { Journal of Korean } \\
\text { Society of Dental } \\
\text { Hygiene }\end{array}$ & $\begin{array}{l}\text { http://www.dbpia.co.kr/Journal/articleDe } \\
\text { tail?nodeId=NODE08990380 }\end{array}$ \\
\hline 31 & SK Park & 2015 & $\begin{array}{l}\text { Educational Needs on National } \\
\text { Oral Health Project among } \\
\text { Community Dental Hygienists }\end{array}$ & Yonsei University & $\begin{array}{l}\text { https://dcollection.yonsei.ac.kr/public_re } \\
\text { source/pdf/000000137689_2020111616 } \\
\text { 1546.pdf }\end{array}$ \\
\hline
\end{tabular}


Table 2. Continued 2

\begin{tabular}{|c|c|c|c|c|c|}
\hline Number & $\begin{array}{l}\text { Main } \\
\text { author }\end{array}$ & $\begin{array}{l}\text { Publication } \\
\text { year }\end{array}$ & Title & Publisher or journal & URL \\
\hline 32 & SS Bae & 2015 & $\begin{array}{l}\text { The standard of Dental } \\
\text { hygienists' clinical job }\end{array}$ & $\begin{array}{l}\text { Korean Dental } \\
\text { Hygienists Association }\end{array}$ & https://www.kdha.or.kr/news/report.aspx \\
\hline 33 & JY Kim & 2016 & $\begin{array}{l}\text { Associations of performance } \\
\text { and perception on preventive } \\
\text { dental care tasks with job } \\
\text { satisfaction among some } \\
\text { dental hygienists }\end{array}$ & Chosun University & $\begin{array}{l}\text { http://chosun.dcollection.net/public_reso } \\
\text { urce/pdf/200000266188_20201116162 } \\
\text { 347.pdf }\end{array}$ \\
\hline 34 & JS Lee & 2016 & $\begin{array}{l}\text { Organization Structure of } \\
\text { Dental Hospital and Job } \\
\text { Analysis of Dental Hygienists }\end{array}$ & Namseoul University & $\begin{array}{l}\text { http://nsulib.dcollection.net/public_resou } \\
\text { rce/pdf/200000196894_202011161625 } \\
\text { 05.pdf }\end{array}$ \\
\hline 35 & SH Chae & 2016 & $\begin{array}{l}\text { Research on dental hygienists' } \\
\text { clinical skill proficiency in } \\
\text { core dental hygiene } \\
\text { competency }\end{array}$ & $\begin{array}{l}\text { Journal of Korean } \\
\text { Society of Dental } \\
\text { HygieneJ }\end{array}$ & $\begin{array}{l}\text { https://doi.org/10.13065/jksdh.2016.16.0 } \\
5.651\end{array}$ \\
\hline 36 & Virtanen & 2016 & $\begin{array}{l}\text { The professional role of a dental } \\
\text { hygienist in Finland - } \\
\text { educators' views }\end{array}$ & $\begin{array}{l}\text { International Journal of } \\
\text { Dental Hygiene }\end{array}$ & https://doi.org/10.1111/idh.12166 \\
\hline 37 & $\mathrm{CH}$ Kim & 2017 & $\begin{array}{l}\text { The awareness of dental } \\
\text { hygienist regarding the content } \\
\text { of clinical practice education } \\
\text { and importance of duty }\end{array}$ & $\begin{array}{l}\text { Journal of Korean } \\
\text { Society of Dental } \\
\text { Hygiene }\end{array}$ & $\begin{array}{l}\text { https://doi.org/10.13065/jksdh.2017.17.0 } \\
6.1067\end{array}$ \\
\hline 38 & SE Moon & 2017 & $\begin{array}{l}\text { A study on the validity of the } \\
\text { range of periodontal treatment } \\
\text { by dental hygienists }\end{array}$ & $\begin{array}{l}\text { Korean Dental } \\
\text { Hygienists Association }\end{array}$ & https://www.kdha.or.kr/news/report.aspx \\
\hline 39 & YH Ha & 2017 & $\begin{array}{l}\text { Mongolian and Korean dentists' } \\
\text { opinions about the role of } \\
\text { dental auxiliary workforce }\end{array}$ & Dankook University & $\begin{array}{l}\text { http://lib.dankook.ac.kr/dcollection/publi } \\
\text { c_resource/pdf/000000188553_202011 } \\
\text { 16163918.pdf }\end{array}$ \\
\hline 40 & SM Bae & 2018 & $\begin{array}{l}\text { Evaluation of Dental Hygienist } \\
\text { Job Validity according to } \\
\text { Judgment Standard of Medical } \\
\text { Practice in Medical Law }\end{array}$ & $\begin{array}{l}\text { Journal of Dental } \\
\text { Hygiene Science }\end{array}$ & $\begin{array}{l}\text { https://doi.org/10.17135/jdhs.2018.18.6. } \\
357\end{array}$ \\
\hline 41 & CY Kang & 2019 & $\begin{array}{l}\text { Dental Hygienist's } \\
\text { Professionalism and Health } \\
\text { Insurance in Korea }\end{array}$ & Yonsei University & $\begin{array}{l}\text { https://dcollection.yonsei.ac.kr/public_re } \\
\text { source/pdf/000000518344_2020111617 } \\
\text { 1430.pdf }\end{array}$ \\
\hline 42 & KJ Kim & 2019 & $\begin{array}{l}\text { A survey of the working scope } \\
\text { understanding between dental } \\
\text { hygienists and chair-side } \\
\text { dental assistants among dental } \\
\text { students in Seoul }\end{array}$ & $\begin{array}{l}\text { Seoul National } \\
\text { University }\end{array}$ & $\begin{array}{l}\text { https://dcollection.snu.ac.kr/public_resou } \\
\text { rce/pdf/000000155246_202011161716 } \\
\text { 00.pdf }\end{array}$ \\
\hline 43 & AO Kim & 2019 & $\begin{array}{l}\text { A comparison of dental } \\
\text { hygienists' legal duties among } \\
\text { nations: Korea, USA and } \\
\text { Canada }\end{array}$ & $\begin{array}{l}\text { Journal of Korean } \\
\text { Society of Oral Health } \\
\text { Science }\end{array}$ & $\begin{array}{l}\text { http://www.riss.kr/search/detail/DetailVi } \\
\text { ew.do?p_mat_type=1a0202e37d52c72d } \\
\text { \&control_no=9f0d6c37c49e012ab3609 } \\
\text { 7776a77e665 }\end{array}$ \\
\hline 44 & JW Yun & 2019 & $\begin{array}{l}\text { Improvement of legal system } \\
\text { regarding the scope of practice } \\
\text { of dental hygienist }\end{array}$ & Dongeui University & $\begin{array}{l}\text { http://dongeui.dcollection.net/public_res } \\
\text { ource/pdf/200000227795_2020111619 } \\
\text { 2350.pdf }\end{array}$ \\
\hline 45 & YK Han & 2019 & $\begin{array}{l}\text { A survey of dentists' opinions } \\
\text { on the performance of dental } \\
\text { hygienists }\end{array}$ & $\begin{array}{l}\text { Journal of Korean } \\
\text { Academy of Dental } \\
\text { Administration }\end{array}$ & $\begin{array}{l}\text { https://www.dbpia.co.kr/journal/articleD } \\
\text { etail?nodeId=NODE09260727 }\end{array}$ \\
\hline 46 & SJ Hwang & 2019 & $\begin{array}{l}\text { Frequency and time of actual } \\
\text { duties of some dental } \\
\text { hygienists }\end{array}$ & $\begin{array}{l}\text { Journal of Korean } \\
\text { Academy of Dental } \\
\text { Administration }\end{array}$ & $\begin{array}{l}\text { https://www.dbpia.co.kr/journal/articleD } \\
\text { etail?nodeId=NODE09260728 }\end{array}$ \\
\hline
\end{tabular}


Table 3. Frequency of Appearance of Literature on Each Task of Dental Hygienists

\begin{tabular}{|c|c|c|c|c|}
\hline \multirow[b]{2}{*}{ Number } & \multirow[b]{2}{*}{ Task } & \multicolumn{3}{|c|}{ Appearance rate $(\%)$} \\
\hline & & Total & $\begin{array}{l}\text { Clinical dental } \\
\text { hygienists }\end{array}$ & $\begin{array}{l}\text { Public dental } \\
\text { hygienists }\end{array}$ \\
\hline 1 & Fluoride application & 67.2 & 61.5 & 100 \\
\hline 2 & Intraoral radiography and panorama taking & 65.4 & 71.8 & 28.6 \\
\hline 3 & Scaling & 65.4 & 74.4 & 14.3 \\
\hline 4 & Sealant & 60.7 & 53.8 & 100 \\
\hline 5 & Patient management and counseling & 56.7 & 64.1 & 14.3 \\
\hline 6 & Tooth-brushing education & 52.2 & 51.3 & 57.1 \\
\hline 7 & Impression taking (alginate) & 50.1 & 56.4 & 14.3 \\
\hline 8 & Temporary crowns making & 47.9 & 53.8 & 14.3 \\
\hline 9 & Setting of temporary crowns & 47.9 & 53.8 & 14.3 \\
\hline 10 & Temporary filling & 45.8 & 51.3 & 14.3 \\
\hline 11 & Diet education & 43.5 & 43.6 & 42.9 \\
\hline 12 & Impressions taking (precision) & 41.4 & 46.2 & 14.3 \\
\hline 13 & Health insurance claim & 41.4 & 46.2 & 14.3 \\
\hline 14 & Oral health education & 39.0 & 33.3 & 71.4 \\
\hline 15 & Prevention of infection in the dental clinic & 37.1 & 43.6 & 0 \\
\hline 16 & Equipment and material management & 37.1 & 43.6 & 0 \\
\hline 17 & Tooth prophylaxis & 37.0 & 38.5 & 28.6 \\
\hline 18 & Removal of excess cement & 37.0 & 41.0 & 14.3 \\
\hline 19 & Materials/document management & 34.9 & 38.5 & 14.3 \\
\hline 20 & Treatment assistance & 34.8 & 35.9 & 28.6 \\
\hline 21 & Taking the medical history & 32.8 & 38.5 & 0 \\
\hline 22 & Gingival cord packing & 32.8 & 38.5 & 0 \\
\hline 23 & $\begin{array}{l}\text { Surgery preparation (extraction and implants, oral and maxillofacial minor } \\
\text { surgery) }\end{array}$ & 32.8 & 38.5 & 0 \\
\hline 24 & Preparation of medical equipment & 32.7 & 35.9 & 14.3 \\
\hline 25 & Teeth whitening & 32.7 & 35.9 & 14.3 \\
\hline 26 & Application of rubber dam & 32.7 & 35.9 & 14.3 \\
\hline 27 & Education to use oral health products & 32.5 & 28.2 & 57.1 \\
\hline 28 & Vital sign check & 30.6 & 35.9 & 0 \\
\hline 29 & Application of the matrix band & 30.6 & 35.9 & 0 \\
\hline 30 & Orthodontic wire ligation & 30.6 & 35.9 & 0 \\
\hline 31 & Model making & 30.5 & 33.3 & 14.3 \\
\hline 32 & Radiographic management & 28.3 & 30.8 & 14.3 \\
\hline 33 & Manegement and disinfection of instruments & 28.3 & 30.8 & 14.3 \\
\hline 34 & Manpower training and management & 28.3 & 33.0 & 0 \\
\hline 35 & Oral examination & 28.2 & 23.1 & 57.1 \\
\hline 36 & Root planing & 26.2 & 30.8 & 0 \\
\hline 37 & Probing periodontal pocket & 26.2 & 30.8 & 0 \\
\hline 38 & Application of orthodontic Elastics & 26.2 & 30.8 & 0 \\
\hline 39 & Fabrication of tooth whitening tray & 24.0 & 28.2 & 0 \\
\hline 40 & Instrument polishing & 24.0 & 28.2 & 0 \\
\hline 41 & Cement mixing & 24.0 & 28.2 & 0 \\
\hline 42 & Restorative material mixing & 24.0 & 28.2 & 0 \\
\hline 43 & Individual tray making & 24.0 & 28.2 & 0 \\
\hline 44 & Medical waste management & 24.0 & 28.2 & 0 \\
\hline 45 & Oral hygiene treatment plan & 23.9 & 25.6 & 14.3 \\
\hline 46 & Emergency treatment in the dental clinic & 21.8 & 23.1 & 14.3 \\
\hline 47 & Intramuscular injection & 21.8 & 25.6 & 0 \\
\hline 48 & Periodical oral health care & 21.8 & 23.1 & 14.3 \\
\hline
\end{tabular}


Table 3. Continued 1

\begin{tabular}{|c|c|c|c|c|}
\hline \multirow[b]{2}{*}{ Number } & \multirow[b]{2}{*}{ Task } & \multicolumn{3}{|c|}{ Appearance rate $(\%)$} \\
\hline & & Total & $\begin{array}{c}\text { Clinical dental } \\
\text { hygienists }\end{array}$ & $\begin{array}{c}\text { Public dental } \\
\text { hygienists }\end{array}$ \\
\hline 49 & Cavity filling & 21.8 & 23.1 & 14.3 \\
\hline 50 & Bite registration & 21.8 & 25.6 & 0 \\
\hline 51 & Assistance of extraction, implants, and oral and maxillofacial minor surgery & 21.8 & 25.6 & 0 \\
\hline 52 & Precautions delivery before and after surgery & 21.8 & 25.6 & 0 \\
\hline 53 & Receiving dental expenses & 21.8 & 25.6 & 0 \\
\hline 54 & Arrangement of equipment & 21.8 & 25.6 & 0 \\
\hline 55 & Topical anesthesia & 19.7 & 23.1 & 0 \\
\hline 56 & Preparation for local anesthesia & 19.7 & 23.1 & 0 \\
\hline 57 & Sensitive tooth treatment & 19.7 & 23.1 & 0 \\
\hline 58 & Care of sedated patients & 19.7 & 23.1 & 0 \\
\hline 59 & Seperating & 19.7 & 23.1 & 0 \\
\hline 60 & Reception of target person & 19.6 & 20.5 & 14.3 \\
\hline 61 & Medical record management & 19.6 & 20.5 & 14.3 \\
\hline 62 & Oral microbial test & 19.5 & 15.4 & 42.9 \\
\hline 63 & Bad breath measurement & 19.5 & 17.9 & 28.6 \\
\hline 64 & Dental caries activity test & 19.5 & 17.9 & 28.6 \\
\hline 65 & Taking cephalometric radiography & 17.4 & 20.5 & 0 \\
\hline 66 & Usage of intraoral camera & 17.4 & 17.9 & 14.3 \\
\hline 67 & Education of stretching head and neck muscles for TMD patients & 17.4 & 20.5 & 0 \\
\hline 68 & Intraoral and extraoral inspection & 17.4 & 20.5 & 0 \\
\hline 69 & Removal of Implant prosthetic screw & 17.4 & 20.5 & 0 \\
\hline 70 & Bonding of orthodontic bracket & 17.4 & 20.5 & 0 \\
\hline 71 & Disinfection of the surgery area & 17.4 & 20.5 & 0 \\
\hline 72 & Education of orthodontic devices usage & 17.4 & 20.5 & 0 \\
\hline 73 & Dental plaque index measurement & 15.2 & 15.4 & 14.3 \\
\hline 74 & Professional dental plaque management & 15.2 & 12.8 & 28.6 \\
\hline 75 & Deciduous teeth extraction & 15.2 & 12.8 & 28.6 \\
\hline 76 & Removal of fixed orthodontic device & 15.2 & 17.9 & 0 \\
\hline 77 & Precaution education after treatment & 15.2 & 17.9 & 0 \\
\hline 78 & Taking facial photograph & 13.1 & 15.4 & 0 \\
\hline 79 & Infiltration anesthesia & 13.1 & 15.4 & 0 \\
\hline 80 & Attachment/removal of periodontal pak & 13.1 & 15.4 & 0 \\
\hline 81 & Electric test of pulp vitality & 13.1 & 15.4 & 0 \\
\hline 82 & Education for dental disease prevention & 13.0 & 12.8 & 14.3 \\
\hline 83 & Making oral health education materials & 12.9 & 7.7 & 42.9 \\
\hline 84 & Suction & 10.9 & 12.8 & 0 \\
\hline 85 & Preparing for mini screw placement & 10.9 & 12.8 & 0 \\
\hline 86 & Stich-out & 10.9 & 12.8 & 0 \\
\hline 87 & Smoking cessation guidance & 10.9 & 12.8 & 0 \\
\hline 88 & Public elderly oral health program & 10.7 & 2.6 & 57.1 \\
\hline 89 & School fluoride mouth-rinsing program & 10.6 & 0 & 71.4 \\
\hline 90 & Public oral health program evaluation & 10.6 & 0 & 71.4 \\
\hline 91 & Computed tomography taking & 8.8 & 10.3 & 0 \\
\hline 92 & Guide to unit chair & 8.8 & 10.3 & 0 \\
\hline 93 & Oral hygiene management record & 8.8 & 10.3 & 0 \\
\hline 94 & Use of light curing unit & 8.8 & 10.3 & 0 \\
\hline 95 & Tooth shade selection & 8.8 & 10.3 & 0 \\
\hline 96 & Dressing after removing healing abutment & 8.8 & 10.3 & 0 \\
\hline
\end{tabular}


Table 3. Continued 2

\begin{tabular}{|c|c|c|c|c|}
\hline \multirow[b]{2}{*}{ Number } & \multirow[b]{2}{*}{ Task } & \multicolumn{3}{|c|}{ Appearance rate $(\%)$} \\
\hline & & Total & $\begin{array}{l}\text { Clinical dental } \\
\text { hygienists }\end{array}$ & $\begin{array}{c}\text { Public dental } \\
\text { hygienists }\end{array}$ \\
\hline 97 & Final polishing after occlusal adjustment & 8.8 & 10.3 & 0 \\
\hline 98 & Radiograph reading & 8.8 & 7.7 & 14.3 \\
\hline 99 & SP crown mounting & 8.8 & 10.3 & 0 \\
\hline 100 & Public oral health program for the disabled & 8.6 & 2.6 & 42.9 \\
\hline 101 & Water fluoridation & 8.5 & 0 & 57.1 \\
\hline 102 & School water fluoridation & 8.5 & 0 & 57.1 \\
\hline 103 & School oral health program & 8.5 & 0 & 57.1 \\
\hline 104 & Other roles related to oral health care & 6.6 & 7.7 & 0 \\
\hline 105 & Management the record and works related to dental technician & 6.6 & 7.7 & 0 \\
\hline 106 & Administrative management & 6.5 & 5.1 & 14.3 \\
\hline 107 & Public oral health promotion program & 6.5 & 2.6 & 28.6 \\
\hline 108 & Establishment of community oral health plan & 6.4 & 0 & 42.9 \\
\hline 109 & Survey of community oral health program & 6.4 & 0 & 42.9 \\
\hline 110 & Public oral health program for the infants & 6.4 & 0 & 42.9 \\
\hline 111 & Removal of exogenous pigmentation & 4.3 & 5.1 & 0 \\
\hline 112 & Conservatory treatment & 4.3 & 5.1 & 0 \\
\hline 113 & Application of liner & 4.3 & 5.1 & 0 \\
\hline 114 & Block out with wax to the interdental area & 4.3 & 5.1 & 0 \\
\hline 115 & Impression coping connection & 4.3 & 5.1 & 0 \\
\hline 116 & Biopsy preparation & 4.3 & 5.1 & 0 \\
\hline 117 & Public sealant program & 4.3 & 0 & 28.6 \\
\hline 118 & Public oral health program for the pregnant women & 4.3 & 0 & 28.6 \\
\hline 119 & Handling laser equipment & 2.2 & 2.6 & 0 \\
\hline 120 & Ultrasound/ physical treatment and hot pack for TMD patients & 2.2 & 2.6 & 0 \\
\hline 121 & Apply adhesive inside the tray & 2.2 & 2.6 & 0 \\
\hline 122 & Interdental cleaning & 2.2 & 2.6 & 0 \\
\hline 123 & Sedation anesthesia $\left(\mathrm{N}_{2} \mathrm{O}\right.$ gas) management & 2.2 & 2.6 & 0 \\
\hline 124 & Inlay setting & 2.2 & 2.6 & 0 \\
\hline 125 & Public oral health center installation & 2.1 & 0 & 14.3 \\
\hline 126 & Administrative work for public oral health program & 2.1 & 0 & 14.3 \\
\hline 127 & Oral health statistics & 2.1 & 0 & 14.3 \\
\hline 128 & Survey for community oral health & 2.1 & 0 & 14.3 \\
\hline 129 & Evaluation of community oral health program & 2.1 & 0 & 14.3 \\
\hline 130 & Public oral health program using vehicles with dental unit-chair & 2.1 & 0 & 14.3 \\
\hline 131 & Customized oral health program with visiting & 2.1 & 0 & 14.3 \\
\hline 132 & School tooth-brushing program & 2.1 & 0 & 14.3 \\
\hline 133 & Public oral health program connected with other health programs & 2.1 & 0 & 14.3 \\
\hline 134 & Public oral disease prevention program & 2.1 & 0 & 14.3 \\
\hline 135 & Other medical treatment program & 2.1 & 0 & 14.3 \\
\hline 136 & Other administrative civil affairs & 2.1 & 0 & 14.3 \\
\hline
\end{tabular}

TMD: temporomandibular joint dysfunction, SP: stainless steel primary.

as planning, execution, managing, and evaluation oral health programs in the community. Of the 67 tasks performed by dental hygienists in public health centers, 33 tasks were not performed by clinical dental hygienists.

\section{Discussion}

Clarifying the tasks of dental hygienists ensured that these tasks are efficiently performed, and a high-quality dental medical service is provided by accurately indicating 
the dental hygienists scope of responsibilities in each job. Objective and scientific evidence and analysis related to the scope of tasks of dental hygienists are required to promote the public oral health. Therefore, this study conducted a systematic review based on the evidence provided in the literature on the tasks of dental hygienists. As a result of the systematic review, dental hygienists performed 136 tasks. The tasks of dental hygienists most discussed in the study were fluoride application, scaling, sealant, and oral health education, such as patient management and counseling, and tooth brushing education.

Among the 136 dental hygienist tasks identified during the literature review, most of the tasks were within the legal scope of their practice, while some were not considered legitimate. In some cases, the tasks that had been performed by dental hygienists were interpreted as illegal tasks, and those that were performed in the past were no longer allowed in current practice. The remarkable development of the dental health industry would have changed the perception of the dental hygienist's job, but the professionalism and autonomy of dental hygienists are still limited. Therefore, an effort must be made to standardize the tasks of a dental hygienist in order to improve the oral health of the public.

If the legality of a dental hygienist's job is not guaranteed, professionalism can be undermined. This will reduce the dental hygienist's job satisfaction and increase the turnover rate. Such situation will become an obstacle in the use of dental hygienists as part of the oral health workforce and will inhibit the improvement of dental treatment. Therefore, the standard tasks of dental hygienist that reflect the current dental medical environment should be established.

Although the boundary for the distinction between root planing and calculus removal is unclear, root planing is not currently recognized as a legal dental hygienist job. Probing, calculus removal, and root planing cannot be separately considered as tests and treatments to maintain periodontal health. Therefore, they must all be recognized as legal tasks. Implant treatment was rapidly popularized as it was recognized as a medical care benefit item covered by the health insurance. Accordingly, the proportion of implant-related tasks of dental hygienists is increasing.
Dental hygienists not only assist with implant surgery, but also perform tasks before and after implant surgery and prosthetics such as removal of implant prosthetic screws, dressing after removal of healing abatement, and removal of sutures. Therefore, it is necessary to recognize the tasks that reflect this situation. In orthodontic treatment, wire removal was included in the tasks of a dental hygienist in the revised Medical Engineers Act, but the interpretations of other tasks remain unclear. Under the guidance of a dentist, dental hygienists are allowed to remove orthodontic brackets, orthodontic elastic application, and seperating. Therefore, other orthodontic treatment items, except for bracket mounting, which greatly influence the results of orthodontic treatment, need to be recognized as part of the dental hygienist's tasks. The application of anesthesia is recognized as the task of a nursing assistant in the general medical field, whereas the application of anesthesia is not recognized as the task of a dental hygienist in the dental field. The administration of anesthesia should also be recognized as a dental hygienist's task, considering the difficulty and risk of the task. With the rapid development of medical technology, the number of equipment used in dentistry is expanding. Various radiological diagnostic imaging devices and equipment, such as laser and ultrasound, are widely used in dental hospitals and clinics. Dental hygienists are responsible for handling and maintaining of these equipments. The tasks of dental hygienists in providing dental medical services are not fixed and constant. Hence, it is necessary to expand the dental hygienists' scope of responsibilities based on the current dental care environment.

The future-oriented tasks of dental hygienists include reading radiographs, prescribing medicines and supplies, examining patients for oral cancer, and managing eating and swallowing disorders. Since radiography is essential in dental hygiene assessment and is used as a tool for imparting oral health education to patients, it can be included as a legal work of dental hygienists under the guidance of a dentist. Prescribing appropriate drugs or supplies for each patient during oral health management should be included in the task of a dental hygienist to provide quality dental services and to improve the oral 
health of visiting patients. In addition, as the oral cancer test kit is continuously developed, it is simpler and easier to use. Dental hygienists can identify oral cancer by conducting an examination during the period of oral health management, hence, the oral cancer test should be part of the task of a dental hygienist.

The difference between the legal duties of dental hygienists prescribed in the current Medical Engineers Act and in the Enforcement Decree and the actual work performed by dental hygienists is that a dental hygienist is an unlicensed medical practitioner and infringes on the public's right to receive a safe oral health care. Therefore, most of the tasks performed by dental hygienists are dental medical practices and should be performed in accordance with the relevant laws and enforcement decrees in order to protect the public health rights to quality and safe oral health care.

Dental hygienists must be oral health care professionals who perform dental hygiene practices with independence, self-determination, and autonomy. Therefore, it is necessary to revise the law according to the frequency, difficulty, importance, and urgency of performing the tasks of a dental hygienist to maintain a high level of professionalism. This does not mean simply expanding the task of a job. Revising the law will improve the efficiency of dental treatment, provide high-quality oral treatment, and enable the public to receive legally safe oral treatment from dental hygienists. Therefore, it is necessary to expand the area and to determine the scope of responsibilities of dental hygienists for the promotion of public oral health.

\section{Notes}

\section{Conflict of interest}

No potential conflict of interest relevant to this article was reported.

\section{Ethical approval}

This study is a review-based study and does not require an IRB review.

\section{Author contributions}

Conceptualization: Ji-Hyoung Han, Sun-Mi Kim, Eunsuk Ahn, Soo-Jeong Hwang, Bo-Ra Kim, Soon-Jeong Jeong. Data acquisition: Ji-Hyoung Han, Sun-Mi Kim, Eunsuk Ahn, Soo-Jeong Hwang, Bo-Ra Kim, Soon-Jeong Jeong. Formal analysis: Eunsuk Ahn, Ji-Hyoung Han, Sun-Mi Kim, Soo-Jeong Hwang, Bo-Ra Kim. Supervision: Ji-Hyoung Han, Eunsuk Ahn, Soo-Jeong Hwang. Writingoriginal draft: Ji-Hyoung Han, Eunsuk Ahn, Sun-Mi Kim, Soo-Jeong Hwang. Writing-review \& editing: Ji-Hyoung Han, Eunsuk Ahn.

\section{ORCID}

Sun-Mi Kim, https://orcid.org/0000-0003-0718-9419

Eunsuk Ahn, https://orcid.org/0000-0002-9404-4826

Soo-Jeong Hwang, https://orcid.org/0000-0003-4725-1512

Soon-Jeong Jeong, https://orcid.org/0000-0002-8959-4663

Bo-Ra Kim, https://orcid.org/0000-0002-3775-280X

Ji-Hyoung Han, https://orcid.org/0000-0003-1613-2879

\section{Acknowledgements}

This study was supported by research fund from, Korean Dental Hygienists Association, 2020.

\section{References}

1. Lee HJ, Shin SJ, Bae SM, Shin BM: A survey of dentists and dental hygienists on dental team composition and work assignments. J Korean Dent Hyg Sci 2: 51-62, 2019.

2. Cho YS: Comparison of curriculums of dental hygiene education programs for B. S degree. J Dent Hyg Sci 5: 251-258, 2005.

3. Moon HJ, Lim SR, Lee GY, Han YS: A study on graduate school choice conviction related in dental hygienics. J Dent Hyg Sci 15: 495-502, 2015. https://doi.org/10.17135/jdhs.2015.15.4.495

4. Retrieved July 1, 2020, from https://rnd.kuksiwon.or.kr/last/ selectLastDetail.do?MENU_ID=C-01-01\&reportno=RE02-1 204-15\&sYear=\&sJssfc_code $=12 \&$ sResearchindexcd $=\&$ res earcherid=\&sRsrchtasknm=\&PAGE_NUM=1\&PER_PAGE =10\&IS_PAGE_NEW_SEARCH=Y\&TOTAL_PAGE=7.pd $\mathrm{f}(2012)$.

5. Kim MH, Lim YH, Lee KA, Kim SJ, Kim YJ: Dental hygiene 
students' awareness of their legal scope and petition for medical personnel. J Korean Acad Dent Admin 6: 36-42, 2018.

6. Jeong KY, Han OS: A study on emotional labor, emotional burnout, turnover intention of dental hygienist. J Dent Hyg Sci 15: 280-286, 2015.

https://doi.org/10.17135/jdhs.2015.15.3.280

7. Choi HA, Bae HS, Cho YS: Effect of gap between expected job and performed job on turnover intention and job satisfaction in clinical dental hygienists. J Dent Hyg Sci 11: 463-469, 2011.

8. Hwang JM, Han JH: Influence of job embeddedness and resilience on turnover intention in dental hygienists. J Dent Hyg Sci 20: 171-177, 2020.

https://doi.org/10.17135/jdhs.2020.20.3.171

9. Kim SY, Park JE, Seo HJ, et al.: NECA's guidance for undertaking systematic reviews and meta-analyses for intervention. National Evidence-based healthcare Collaborating Agency, Seoul, 2011. 287 p.

10. Moher D, Liberati A, Tetzlaff J, Altman DG; PRISMA Group: Preferred reporting items for systematic reviews and meta-analyses: the PRISMA statement. PLoS Med 6: e1000097, 2009.

https://doi.org/10.1371/journal.pmed.1000097 